\title{
La propriété immobilière des femmes musulmanes de Jérusalem (1831-1841)
}

\section{Entre la reconnaissance et la spoliation}

\author{
Musa Sroor \\ Birzeit University, History and Archeology Department \\ msroor@birzeit.edu
}

\begin{abstract}
This study shows the important place of Muslim women in the civil society of Jerusalem during the period 1831-1841 (i.e., the Egyptian period) via their right to own and use property according to their own volition. I have analyzed these issues according to the following questions: What was the attitude of local society with respect to Muslim women's rights to dispose of their assets as they saw fit? Was this accepted by local society or not?

As revealed by the documents, whereas Muslim families in Jerusalem generally accepted the principle of the right of ownership and use of property by women, nonetheless, this study shows that numerous families in Jerusalem did not, in reality, accept these rights.
\end{abstract}

\section{Keywords}

Jerusalem - women - real estate - Ottoman Jerusalem - property law - law of the use of property - women and law - law and inheritance - socioeconomic history

\section{Résumé}

Cette étude a tenté de démontrer la place importante de la femme dans la société civile de Jérusalem durant la période 1831-1841, grâce à son droit de propriété et à la liberté d'en disposer. Nous avons traité cette question à partir des réponses aux questions suivantes : Quelle était alors l'attitude de la société locale vis-à-vis du droit des femmes du libre usage de leur argent? Est-ce qu'elle acceptait facilement ou pas? 
Le milieu familial musulman à Jérusalem acceptant le principe d'un droit de propriété et le droit de l'usage ; mais dans plusieurs cas comme nous avons bien montré qu'il y avait de nombreuses familles jérusalémites n'acceptant pas le principe d'un droit de propriété ni le droit de l'usage.

\section{Mots clés}

Jérusalem ottoman - femmes - propriété immobilière - droit et propriété - droit de l'usage de propriété - femmes et droit - droit et héritage - histoire socioéconomique

A travers l'histoire, les hommes et les femmes ont travaillé en vue de développer la ville de Jérusalem tant sur le plan architectural que charitable et culturel. En cela, la création et l'utilisation de la propriété foncière jouaient un rôle prédominant. A cet égard, les femmes ont laissé des traces dans l'histoire de Jérusalem comme pour d'autres villes du monde arabo-musulman. A titre d'exemple, en 755 de l'hégire 1354, une femme du nom d'Âghal Khâtûn bt. Shams al-Dîn al-Baghdâdiyya a fondé à Jérusalem un waqf ${ }^{1}$ dont le bénéficiaire est une école coranique (madrasa) à laquelle elle donne son nom ${ }^{2}$. En 964 de l'hégire 1557, Khasseki Sultan (Hürrem Sultan) ${ }^{3}$, femme du sultan Soliman le Magnifique, a créé dans la même ville un immense complexe soutenu par un waqf charitable connu sous le nom de takiyya ${ }^{4}$ en faveur d'une école coranique ainsi que de cinquante-cinq chambres pour accueillir gratuitement des voyageurs et aussi une immense cuisine où se préparaient des centaines de repas distribués gratuitement aux pauvres, aux voyageurs et aux riverains de la mosquée al-Aqșâ. Cette takiyya avait un four (furn) où se préparaient chaque jour 2 ooo galettes de pain (fadûlas) ${ }^{5}$.

Les deux femmes en question n'étaient pas originaires de Jérusalem mais leurs motifs de créer leurs waqfs se liaient à l'importance religieuse de la Ville

1 Pour connaître la définition de waqf, voir Khalâf, 1946, p. 14.

2 'Abd al-Mahdî, 1981, p. 60 ; Sroor, 2010, p. 161.

3 Les sources sont contradictoires sur la date de la construction de la takiyya. Selon Abû Bakr, elle a été fondée en 959 /1551: Abû Bakr, 1996, p. 438 mais selon Stephan, elle a été fondée en mai $155^{2}$ : Stephan, 1944, p. 170. Selon l'acte de fondation (waqfiyya), elle a été établie au mois de Sha'bân 964 / mai 1557 : sijill 270, p. 18-22.

4 Complexe également appelée 'imâra puisque cette fondation caritative est au service des pauvres auxquels elle fournissait des repas mais aussi au service de gens plus aisés qui habitaient les environs de ce complexe.

5 Sijill 270, 1557/964, 18-27 ; Stephan, 1944, p. 172-3 ; Sroor, 2010, p. 167-9. 
Sainte. A cet égard, une question se pose : quels étaient le rôle et l'action des femmes originaires de Jérusalem?

Dans les actes de registres (sijill) du tribunal religieux sharîa (mahkkamas) de Jérusalem ${ }^{6}$, source d'information extrêmement riche concernant le rôle des femmes dans la société à l'époque ottomane, on trouve des actes traitant de l'héritage mais aussi du waqf, des contrats de vente et d'achat ainsi que des litiges sur les droits de propriété. On remarque la présence importante des femmes dans ces documents. Rappelons que l'islam n'accorde pas l'égalité de l'héritage entre les hommes et les femmes; toutefois, remarquons que les hommes qui héritent du double des femmes doivent subvenir aux besoins de la famille. De plus, dans la coutume traditionnelle locale qui perdure encore, pour avoir droit à sa part d'héritage, la femme doit être d'une moralité irréprochable sauf cas exceptionnel ${ }^{7}$. Les documents des registres du tribunal montrent que les femmes possédaient par la voie de l'héritage des propriétés immobilières et qu'elles en avaient libre usage (pour les vendre, les mettre en waqf, etc.).

\section{La méthodologie de cette étude}

Quelle était alors l'attitude de la société locale pour la période étudiée, 1831-41, vis-à-vis des droits des femmes quant au libre usage de leurs propriétés? Le libre arbitre exercé par des femmes était-il chose acquise ou y a-t-il eu une résistance à cela et par qui, dans quelles circonstances? Dans ce cas, comment exprimait-elle l'opposition aux femmes qui exerçaient leurs droits de propriété?

Nous avons choisi d'étudier des exemples provenant des actes du tribunal religieux de Jérusalem durant la période de 1831 à 1841, une période caractérisée en Palestine par la domination des provinces égyptiennes ottomanes dirigées par Muhammad Ali Pacha, gouverneur de l'Egypte ${ }^{8}$. Il y a deux raisons pour ce choix de période. La première est de l'ordre méthodologique dans la mesure où cela nous permet d'encadrer le sujet d'étude sur dix ans (chaque registre étudié couvre une année). Les registres, n'étant pas indexés, nous ont obligés de systématiquement extraire les actes qui concernaient la propriété des femmes à Jérusalem. Nous avons établi ces données en plusieurs tableaux. A partir de

6 Pour des informations sur cette source, voir Sroor, 2005, p. 80-6; Manna', 1986; Salameh, 2000.

7 Pour des informations sur cette question, voir al-Mar'a al-Filațstîniyya wa-'l-Mîrâth qui est publié au Markaz al-mar'a li-'l-irshâd al-qânûnî wa-'l-ijtimâ'î, Filațstîn, 2014.

8 Pour cette question, voir Safi, 2004. 
ces tableaux, nous avons analysé les données pour obtenir des résultats précis grâce à une méthode à la fois qualitative et quantitative. La seconde raison pour le choix de cette période est le fait qu'elle constitue une tranche chronologique distincte de l'époque ottomane en Palestine (1516-1917).

Rappelons que cette période dite égyptienne est une nouvelle étape de l'histoire de Jérusalem ottomane : parmi les nombreuses modifications instituées pendant la décennie de 1831 à 1841, signalons un décret concernant l'égalité entre musulmans et non musulmans ainsi que des décisions modifiant les propriétés foncières ${ }^{9}$. Signalons ces deux éléments, notamment, le premier qui était destiné à contourner la domination des élites musulmanes locales afin de créer d'autres cercles de pouvoir. Dans la lignée de cette politique, Muhammad Ali a décidé de créer des conditions plus favorables pour l'accueil des étrangers, i.e. des non-ottomans avec l'objectif d'ouvrir la région vers l'extérieur ${ }^{10}$. A notre avis, le contact avec l'étranger a probablement ouvert des perspectives autres aux habitants de Jérusalem, y compris, un élargissement des droits à la propriété des musulmanes.

L'importance de notre étude est qu'elle traite d'une question «ouverte» dans la mesure où la situation concernant l'accès des femmes aux droits de propriété se pose encore maintenant non seulement à Jérusalem mais dans tout le monde musulman ainsi que, au demeurant, ailleurs dans le monde. Et cela malgré toutes les évolutions internationales concernant le droit des femmes dans le monde.

Nous allons traiter ce sujet à partir de trois axes :

Le premier : le droit des femmes et la propriété ;

le deuxième : le droit des femmes à disposer de leurs biens fonciers;

le troisième : la position des hommes par rapport au droit de propriété des femmes et de leur droit à disposer de leurs biens fonciers.

Nous allons traiter ces questions à partir de 217 documents enregistrés dans les sijills qui couvrent la période de notre étude, 1831-1841. Nous avons divisé ces documents en cinq catégories :

- actes traitant de l'héritage des femmes;

- waqfs fondés par des femmes;

- contrats de vente de propriété foncière effectués par des femmes;

- contrats d'achat de propriété foncière effectués par des femmes;

- litiges sur les droits de propriété des femmes.

$9 \quad$ Ibid., p. 160-4.

$10 \quad$ Ibid., p. 154-6o. 
Le tableau $\mathrm{n}^{\circ} 1$ recense ces cas :

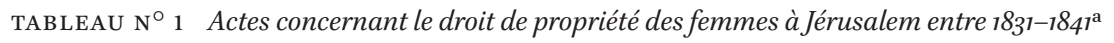
Division selon les années

\begin{tabular}{llllll}
\hline Année & $\mathrm{N}^{\circ}$ des cas & $\mathrm{N}^{\circ}$ des contrats & $\mathrm{N}^{\circ}$ des contrats & $\mathrm{N}^{\circ}$ des & $\mathrm{N}^{\circ}$ des cas de \\
d'héritage & d'achats des & de vente des & fondations & litiges sur les \\
& biens & biens & deswaqfs & $\begin{array}{l}\text { droits de propriété } \\
\text { des femmes }\end{array}$ \\
& & & &
\end{tabular}

\begin{tabular}{rrrrrr}
\hline 1831 & 9 & 5 & 7 & 0 & 2 \\
1832 & 6 & 3 & 3 & 0 & 0 \\
1833 & 9 & 3 & 7 & 0 & 0 \\
1834 & 8 & 6 & 8 & 1 & 0 \\
1835 & 3 & 7 & 6 & 0 & 0 \\
1836 & 2 & 3 & 5 & 0 & 3 \\
1837 & 7 & 6 & 14 & 1 & 6 \\
1838 & 1 & 11 & 12 & 0 & 3 \\
1839 & 0 & 6 & 14 & 0 & 1 \\
1840 & 7 & 3 & 6 & 0 & 2 \\
1841 & 2 & 3 & 7 & 0 & 0 \\
Total & 54 & 56 & 88 & 2 & 17 \\
& & & & & 0 \\
\hline
\end{tabular}

a Sijill 316, 1247-1248 / 1831-1832 ; Sijill 317, 1248-1249 / 1832-1833 ; Sijill 318, 1249-1250 / 18331834 ; Sijill 319, 1250-1251 / 1834-1835 ; Sijill 320, 1251-1252 / 1836-1837 ; Sijill 321, 1252-1253 / 1836-1837 ; Sijill 322, 1253-1254 /1837-1838 ; Sijill 323, 1254-1256 / 1839-1840 et Sijill 324, 12561257 / 1840-1841.

\section{Premier exemple : le droit des femmes et la propriété}

Durant la période de cette étude, nos documents montrent que les femmes hiérosolymites ont possédé des propriétés foncières, soit à Jérusalem intramuros, soit extra-muros. L'origine de ces propriétés correspond à leur droit en islam de posséder pleinement une propriété (milk). En effet, l'islam donne à la femme le droit d'hériter en pleine propriété de ses proches: de son père, elle reçoit la moitié de ce qui revient à son frère et de son mari, elle reçoit un huitième de l'héritage de celui-cil ${ }^{11}$. Les femmes ont, de par le $f i q h$, la jurisprudence

11 Le Coran, Sûrat al-Nisâ', ayâ 12. 
musulmane, le droit d'acheter des biens avec leur propre argent. Nos documents montrent que la possession de propriété par des femmes hiérosolymites ait été acquise par deux moyens : l'héritage et l'achat.

\section{$1 \quad$ Le fiqh et l'héritage}

Pour la période étudiée, nous avons trouvé cinquante-quatre cas provenant des documents d'inventaires après décès, les tarikât, inscrits dans les sijills qui témoignent du fait que les parents et les maris avaient agi en accord avec le figh concernant l'héritage à l'égard de leurs filles et leurs femmes. Le tableau suivant recense cinq cas d'héritage. On n'a trouvé aucun document qui porte évidence d'une interdiction faite à une femme vis-à-vis de son héritage que ce soit en provenance de son mari ou de son père. Or ce constat n'assure pas bien entendu une situation éventuelle à Jérusalem où des individus ne respectent pas le droit des femmes quant à leur héritage mais on pourrait peut-être expliquer ceci par le rôle du juge du tribunal religieux dont la responsabilité est d'assurer une adhérence aux règles du droit musulman. En effet, après la mort d'un individu, le juge devait effectuer le partage légal des successions entre les héritiers, hommes et femmes.

Les inventaires étudiés ici sont construits sur un modèle relativement uniforme: en préambule est indiqué le nom du défunt, son titre, son métier, son lieu de résidence, les noms et qualités des ayants droit et des personnes qui les représentent et sollicitent l'intervention du juge. Ensuite, on procède à l'énumération chiffrée de l'actif, à savoir les biens mobiliers et immobiliers. Enfin, le montant net de la succession est partagé entre les héritiers. Dans la totalité des cinquante-quatre cas étudiés ici, le juge a bien suivi la procédure de l'héritage du droit musulman, soit deux parts pour le garçon, une part pour la fille et une huitième pour la femme du défunt. Au cas où le défunt avait deux femmes, toutes les deux partagent la même part, une huitième, comme indique le cas $\mathrm{n}^{\circ} 3$ dans le tableau $\mathrm{n}^{\circ} 2$.

Le tableau $\mathrm{n}^{\circ} 2$ montre cinq cas comme exemples.

\section{$2 \quad$ Le droit d'achat}

Grâce à notre recherche dans les dix sijills qui couvre la période de notre étude, nous avons trouvé cinquante-six contrats d'achat des biens immobiliers effectués par ou pour des femmes inscrites dans ces registres sans remarque d'opposition de la part de ses proches. Dans la plupart des cas, ses proches comme leurs maris ou leurs frères les ont accompagnées au tribunal en tant que leurs représentants légaux (wakîls). Le tableau $\mathrm{n}^{\circ} 3$ ci-dessous recense quelques exemples de ces cas. Le tableau indique le nom de l'acheteuse, le nom du vendeur / de la vendeuse, le nom du représentant, le type de bien, le prix et la date 
TABLEAU N ${ }^{\circ} 2 \quad$ Exemples d'héritage des femmes hiérosolymites entre 1831-1841

\begin{tabular}{|c|c|c|c|}
\hline $\mathrm{N}^{\circ}$ & Nom de la femme ayant hérité & $\begin{array}{l}\text { Nom de l'homme décédé } \\
\text { (mari ou père) }\end{array}$ & Date \\
\hline 1 & Maḥbûba Mubârak & Sha'bân al-Mughrabî & $1247 / 1831^{\mathrm{a}}$ \\
\hline 2 & 'Â'isha Salîm Ấghâ & Abû Su'ûd al-Dajânî & $125^{1} / 186^{b}$ \\
\hline 3 & $\begin{array}{l}\text { Fâțimâ Najîm et } \\
\text { Sakînâ Khalîl 'Ishâ }\end{array}$ & Aḥmad Khalîl al-Ni'âjî & $125^{2} / 1837^{\mathrm{c}}$ \\
\hline 4 & $\begin{array}{l}\text { Sukayba 'Abd al-Qâdir } \\
\text { al-'Alamî }\end{array}$ & Dâwûd Afandî al-Dajânî & $1254 / 183^{d}$ \\
\hline 5 & $\begin{array}{l}\text { 'Â'isha Ahmmad al-Rifâ'î, } \\
\text { ancienne esclave du Khayzarân }\end{array}$ & 'Umar al-Khalîlî & $1256 / 1840^{\mathrm{e}}$ \\
\hline
\end{tabular}

a Sijill 315 , début Rajab 1247 / 6 décembre 1831, p. 132.

b Sijill 320, 15 Shawwâl 1251 / 3 février 1836, p. 34-35.

c Sijill 321, 10 Dhû al-Qa' da 1252 / 16 février 1837, p. 34-35.

d Sijill 322, 21 Jumâdâ I 1254 / 12 août 1838, p. 67 .

e Sijill 323 , début Dhû al-Qa dâ 1256 / 25 décembre 1840, p. 58 .

de chaque contrat. Dans notre corpus pour 1831-1841, la quantité de ces cas est, en effet, très importante : elle nous donne des informations détaillées concernant la femme hiérosolymite qui possède de moyens monétaires pour acheter des biens fonciers et ensuite les enregistrer sous son propre nom auprès du tribunal.

Ces exemples montrent que les femmes ont acheté des biens non pour établir des commerces mais, en revanche, elles se procuraient des petites parts des propriétés qui leurs étaient vendues et qui servaient à augmenter leur capital. Ces exemples révèlent que les propriétés foncières possédées par plusieurs personnes existaient en raison du système de l'héritage en islam où les biens d'un défunt sont divisés entre les héritiers. Nous constatons seulement sept cas parmi les cinquante-six exemples étudiés où la femme avait acheté intégralement un bien au lieu d'une part comme est la situation dans la majorité des cas. Les sept exemples étudiés ici comprennent six maisons (dârs) et un potager (hâkûra). Selon les informations disponibles, il faut signaler que la plupart des femmes dans les sept cas signalés ne sont pas issues de familles notables de Jérusalem. Elles descendent de la souche moyenne et, par ailleurs, ne sont pas originaires de Jérusalem. Autrement dit, elles sont issues de l'immigration des individus partis à Jérusalem pour y installer comme est le cas pour la famille 
al-Mașrî, originaire d'Egypte, la famille al-Jabâlî, originaire de Gaza (cf. le cas $\mathrm{n}^{\circ} 4$ dans le tableau $\mathrm{n}^{\circ}$ 3) et d'autres familles comme les Salamûnî et les Tikrûrî

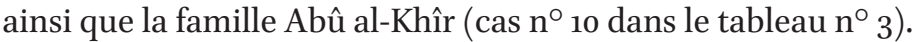

A partir d'une étude au sujet des noms des acheteuses et des vendeurs ou vendeuses, nous constatons que dans la plupart des cas, il n'y a aucune relation familiale entre les deux, seulement dix exemples sur cinquante-six révèlent des liens familiaux entre vendeur(e) et acheteuse. Parmi ces exemples, le cas $\mathrm{n}^{\circ} 1$ dans le tableau $\mathrm{n}^{\circ} 3$ parle d'une femme qui a acheté un bien de sa mère ainsi que le cas $\mathrm{n}^{\circ} 2$ où une femme a acheté une propriété de son mari ou encore le cas $n^{\circ} 6$ où une femme a acheté de son frère.

TABLEAU N ${ }^{\circ} 3$ Exemples d'achat des biens fonciers par des femmes hiérosolymites entre $1831-1841$

\begin{tabular}{|c|c|c|c|c|c|c|}
\hline $\mathbf{N}^{\circ}$ & $\begin{array}{l}\text { Nom de } \\
\text { l'acheteuse }\end{array}$ & $\begin{array}{l}\text { Nom du } \\
\text { vendeur(e) }\end{array}$ & $\begin{array}{l}\text { Nom du } \\
\text { procureur }\end{array}$ & Type de bien & Prix & Date \\
\hline 1 & Fâțima Kîlûn & Sa mère Șâliḥa & $\begin{array}{l}\text { Son mari } \\
\text { Qâsim }\end{array}$ & $\begin{array}{l}1 \text { qîrât } t^{\mathrm{a}} / d \hat{a} r^{\mathrm{b}} \\
\text { (maison) }\end{array}$ & $\begin{array}{l}100 \\
\text { qirsh }\end{array}$ & $\begin{array}{l}1247 / \\
1831^{\mathrm{c}}\end{array}$ \\
\hline 2 & Nafîsa Hâshim & $\begin{array}{l}\text { Muhammad } \\
\text { al-Khâlidî }\end{array}$ & $\begin{array}{l}\text { Son mari } \\
\text { (illisible) }\end{array}$ & $1 d \hat{a} r$ & 1700 & $\begin{array}{l}1247 / \\
183^{\mathrm{d}}\end{array}$ \\
\hline 3 & Ḥasna al-Ṣayyâd & $\begin{array}{l}\text { Sulaymân } \\
\text { 'Ațiyya }\end{array}$ & Son mari 'Alî & $\begin{array}{l}\text { Le droit de } \\
\text { muzâra'a } \\
\text { (affermage) d'un } \\
\text { terrain }\end{array}$ & 300 & $\begin{array}{l}1249 / \\
1833^{\mathrm{e}}\end{array}$ \\
\hline 4 & $\begin{array}{l}\text { Șafiyya et Husun } \\
\text { al-Jabâlî }\end{array}$ & $\begin{array}{l}\text { Muhammad } \\
\text { al-Bawwâb }\end{array}$ & $\begin{array}{l}\text { Juge de } \\
\text { Jérusalem }\end{array}$ & $\begin{array}{l}\text { Totalité d'une } \\
\text { hâkûra }\end{array}$ & 300 & $\begin{array}{l}125^{\circ} / \\
1834^{\mathrm{f}}\end{array}$ \\
\hline 5 & $\begin{array}{l}\text { Maḥbûba et sa } \\
\text { sœur Fațûma } \\
\text { Muhammad } \\
\text { al-Khâlidî }\end{array}$ & $\begin{array}{l}\text { Khadîja 'Umar } \\
\text { al-Jâ'ûnî }\end{array}$ & $\begin{array}{l}\text { Son mari } \\
\text { Muhamammad } \\
\text { al-Khâlidî }\end{array}$ & 4.5 qîrâțs / dâr & 1400 & $\begin{array}{l}125^{\circ} / \\
1834^{\mathrm{g}}\end{array}$ \\
\hline 6 & $\begin{array}{l}\text { Asmâ Luṭî } \\
\text { Hujîij }\end{array}$ & $\begin{array}{l}\text { Son frère } \\
\text { 'Uthmân }\end{array}$ & $\begin{array}{l}\text { Khalîl Bîk } \\
\text { al-Turjumân }\end{array}$ & $\begin{array}{l}1 \text { qîrât / dâr et } 2 \\
\text { dukkâns et } 3 \\
\text { qîrâts / hâkkûra }\end{array}$ & 1200 & $\begin{array}{l}1253 / \\
1837^{\mathrm{h}}\end{array}$ \\
\hline 7 & $\begin{array}{l}\text { Nafîsa } \\
\text { al-Salamûnî }\end{array}$ & Husayn al-Khalî & $\begin{array}{l}\text { lîMușțafâ } \\
\text { al-Sulaymî }\end{array}$ & 1 dâr & 1500 & $\begin{array}{l}1254 / \\
1838^{\mathrm{i}}\end{array}$ \\
\hline 8 & Șâliḥ al-Tikrûrî & Jabr al-Farrân & 1 & $1 d \hat{a} r$ & 500 & $\begin{array}{l}1254 / \\
188^{\mathrm{j}}\end{array}$ \\
\hline
\end{tabular}




\begin{tabular}{|c|c|c|c|c|c|c|}
\hline $\mathrm{N}^{\circ}$ & $\begin{array}{l}\text { Nom de } \\
\text { l'acheteuse }\end{array}$ & $\begin{array}{l}\text { Nom du } \\
\text { vendeur(e) }\end{array}$ & $\begin{array}{l}\text { Nom du } \\
\text { procureur }\end{array}$ & Type de bien & Prix & Date \\
\hline 9 & Hasûna al-Khalîlî & $\begin{array}{l}\text { Umar } \\
\text { al-Daqqâq }\end{array}$ & $\begin{array}{l}\text { Husayn } \\
\text { al-Mughrabî }\end{array}$ & 23 qîrâts / dâr & $235^{2}$ & $\begin{array}{l}1255 / \\
1839^{k}\end{array}$ \\
\hline 10 & $\begin{array}{l}\text { Khadîja Abû } \\
\text { al-Khîr }\end{array}$ & Maḥmûd Rashqî / & & $1 d \hat{a} r$ & 800 & $\begin{array}{l}1256 / \\
1840^{1}\end{array}$ \\
\hline
\end{tabular}

a Vingt-quatre qîrâts composent la totalité d'une propriété, donc vingt-quatre qîrâț voudraient dire $100 \%$ d'un bien, douze qîrâț indiquent 50\% d'un bien, etc.

b Certaines recherches indiquent que le dâr est «un bâtiment indépendant, bien délimité, composé de plusieurs espaces au rez-de-chaussée et à l'étage, qui s'organisent autour d'une cour; il regroupe donc un espace découvert, la cour, pour le délassement et des espaces bâtis propres à y loger » : Pascual, 1990, p. 392.

c Sijill 315, début Șafar 1247 / 12 juillet 1831, p. 9.

d Sijill 316, fin Dhû al-Qa da 1247 / 1 mai 1832, p. 25.

e Sijill 317, Rabî' I 1249 / août 1833, p. 158.

f Sijill 319, fin Rabî'II 1250 / 14 septembre 1834, p. 19.

g Sijill 319, milieu Muharram 1250/24 mai 1834, p. 103.

h Sijill 321, fin Sha bân 1253 / 29 novembre 1837, p. 199-200.

i Sijill 322, milieu Jumâdâ II 1254 / 5 septembre 1838, p. 81 .

j Sijill 322, début Sha'bân 1254 / 20 octobre 1838, p. 124.

k Sijill 323, début Ramaḍân 1255 / 8 novembre 1839, p. 47.

l Sijill 324, 20 Sha'bân 1256 / 17 octobre 1840, p. 73 .

\section{Deuxième exemple : le droit des femmes à disposer de leurs biens fonciers, $1831-1841$}

L'examen des registres du tribunal religieux pour la période de cette étude nous a permis de constater deux possibilités pour des musulmanes de Jérusalem concernant la manière par laquelle elles puissent disposer de leurs biens fonciers. La première concerne le droit accordé aux femmes pour la mise en waqf de leurs propriétés tandis que la deuxième porte sur la vente de leurs biens.

\section{$1 \quad$ Les biens mis en waqf par des musulmanes}

Dans cette section, nous allons traiter des biens mis en waqf par des musulmanes à Jérusalem durant la période de cette étude à partir des informations apportées aux questions suivantes. Combien de biens ont-elles mis en waqf? S'agissait-il de biens établis en faveur d'un waqf charitable (waqf khayrî), 
familial (waqf ahlî / dhurrî) ou mixte (waqf mushtarak)? Quel était le statut social des fondatrices? Comment avaient-elles acquis ces biens : par la voie d'héritage, d'achat ou autre? Quant à l'emplacement dans la ville de Jérusalem de ces propriétés mises en waqf par ces femmes, se trouvaient-elles rassemblées dans un quartier ou disséminées dans la ville?

Pour répondre à ces questions, nous analysons ici deux actes de fondations de waqf établis par des musulmanes et enregistrés au tribunal religieux de Jérusalem entre $183^{1}$ et 1841. Pour cette période, nous avons seulement trouvé treize actes de waqfs inscrits dans les registres parmi lesquels deux qui ont été fondés par des musulmanes. Tout d'abord, il faut signaler que ces deux waqfs sont des fondations familiales $(\text { ahlî } / \text { dhurrî })^{12}$, aucun de treize actes qui portent sur les waqfs ne fait mention de la création d'un waqf khayrî (charitable $)^{13}$. Pour faire une comparaison, nous pouvons signaler que la période de la deuxième moitié du XIX ${ }^{\mathrm{e}}$ siècle avait été moins propice que la première moitié du même siècle pour la création de waqfs musulmans à Jérusalem car, selon nos propres recherches, seulement douze waqfiyyas (acte de création d'un waqf) ont été inscrits dans les registres des cadis de Jérusalem pendant la deuxième moitié du XIX ${ }^{\mathrm{e}}$ siècle. Nous avons pu constater, à la lecture de ces actes de waqfs, la présence importante des musulmanes parmi les fondateurs de waqf à Jérusalem et leurs rôles dans la création des waqfs, à savoir cinq waqfs sur un total de douze, soit environ $40 \%$ qui ont été créé par des femmes ${ }^{14}$.

A partir de ce constat, nous pouvons supposer que les deux waqfs créés par les musulmanes durant la période de notre étude ont été établis pour des intérêts privés. Les fondatrices potentielles ont pu décider de mettre leurs biens en waqfs dhurrî car dans ce cas, elles pouvaient être à la fois gestionnaires et bénéficiaires du waqf mais aussi gardaient leurs biens intacts en les protégeant d'un transfert hors du statut de waqf. Les éléments suivants sont extraits de l'analyse de ces documents qui étayent cette hypothèse.

Tout d'abord, ces fondatrices ont opté pour la gestion personnelle de leurs biens pendant toute leurvie. A leur décès, elles laissaient des recommandations

12 Le waqf ahlî / dhurrî est celui où le fondateur / la fondatrice pouvait se désigner en tant que bénéficiaire ou bien ses descendants ou encore une ou plusieurs personnes de son choix, en fixant leurs parts de revenus généré par les propriétés appartenant au waqf ainsi que l'ordre de succession selon des règles juridiques précises. Voir Deguilhem, 1986, p. 69 ; Deguilhem, 1995a, p. 16 ; Deguilhem, 1995b, p. 203 ; Denoix, 1995, p. 32 ; Saliba, 2004, p. 109.

13 Le waqf khayrî est celui où le fondateur a désigné comme bénéficiaire une œuvre publique, caritative, religieuse ou d'intérêt général. Voir Yediyildiz, 1985, p. 13, 14; Deguilhem, 1995a, p. 45 ; Ben Achour, 1992, p. 52 ; Sroor, 2010, p. 110.

14 Sroor, 2010, p. 200-4. 
à leur famille et à leurs descendants qui héritaient cette fonction, génération après génération. A titre d'exemple, Ammûna al-Qaș̣âș s'est présentée à la fin du mois de Sha'bân 1250 / 1 janvier 1835 devant le conseil légal du tribunal religieux de Jérusalem pour confirmer la constitution en waqf des biens suivants : la totalité de la part indivise qui représente huit qîrâț sur un total de vingt-quatre qîrâț de la maison (dâr) sise à mahallat Bâb al-'Âmûd (le quartier de la porte de Damas) à Jérusalem intra-muros. Dans le document, Ammûna a précisé qu'elle sera la gestionnaire de son waqf. A son décès, la gestion sera transmise à son neveu Ḥusayn et, après lui, aux « «plus sages de ses enfants (al-arshad fa al-arshad) qui partageront la gestion ${ }^{15}$.

Les deux fondatrices ont initialement établi leurs waqfs en leur propre faveur, puis au profit des membres de leurs familles. La fondatrice Ammûna établit son waqf pour elle-même, de son vivant. A sa mort, les revenus de son waqf seront transmis à son neveu Ḥusayn. Au décès de cette personne, les bénéficiaires de ce waqf seront les fils de Husayn selon le partage imposé par la jurisprudence musulmane, soit deux parts pour le garçon, une part pour la fille ${ }^{16}$.

A l'étude de ces cas, nous remarquons que ces femmes possédaient des biens fonciers qu'elles avaient hérités et transformés en waqfs: il faut noter que le document précise bien cette voie d'acquisition de propriété. En outre, signalons que ces femmes possédaient ces biens fonciers en partage avec des proches qu'elles ont transformés en waqfs : c'est-à-dire qu'elles avaient mis en waqf leur part seulement du bien en question.

Enfin, nous pouvons dire que ces femmes ont mis leurs biens en waqf non pour une question charitable mais pour bien profiter de ces biens pendant leur vie et pour protéger ces propriétés contre la spoliation car le statut du waqf les protège a priori d'une confiscation.

\section{$2 \quad$ Le droit des femmes hiérosolymites de vendre leurs biens fonciers}

Nous avons examiné le droit des femmes hiérosolymites quant à la vente de leurs biens fonciers à partir de quatre-vingt-huit actes de contrat de vente des propriétés inscrites dans les registres des cadis de Jérusalem pour la période de notre étude. Citons quelques exemples détaillés dans le tableau $\mathrm{n}^{\circ} 4$ suivant. Nous avons recensé seulement les contrats où la vente est effectuée par une femme habitant Jérusalem. Ce tableau nous indique le nom de la vendeuse, le nom de l'acheteur, le type de bien, l'origine de la propriété, le prix et la date. L'analyse de quatre-vingt-huit actes de contrat de vente nous donne les éléments suivants.

\footnotetext{
15 Sijill 319, fin Sha'bân 1250 / 1 janvier 1835, p. 59 .

16 Idem.
} 
Au premier abord, remarquons la présence active de la femme hiérosolymite dans le marché foncier durant cette période, soit par la vente des biens fonciers, soit par l'achat. Le nombre de contrats de vente (quatre-vingt-huit) est bien supérieur par rapport aux contrats d'achat (cinquante-six). Ce nombre nous donne une indication que la femme vend plus ses biens qu'elle n'en achète. A notre avis, cela s'explique par deux facteurs: le premier concerne le besoin d'argent alors que le deuxième porte sur la pression exercée par ses proches de vendre ses biens en raison de la complexité du système de l'héritage et la difficulté, par conséquent, de profiter de ses biens hérités, soit pour une utilisation personnelle, soit pour les mettre en location. Nous constatons que seulement sept parmi les quatre-vingt-huit contrats concernent une situation où la vendeuse a possédé la totalité du bien vendu. Parmi ces sept biens vendus, il y en a deux où les vendeuses les possédaient par la voie de l'achat tandis que les cinq autres par l'héritage. Quant aux autres exemples, les femmes ont vendu leurs parts des biens hérités.

Après l'analyse de ces contrats et après avoir examiné les origines des propriétés indiquées dans ces contrats, nous remarquons que ces biens sont divisés comme suit: quarante-huit biens possédés par l'héritage provenant des pères de ces femmes; cinq biens possédés par l'héritage de leurs mères; vingt biens possédés par l'héritage de leurs maris; quatre biens possédés par l'héritage de leurs frères; neuf biens obtenus par l'achat et trois biens possédés par d'autres moyens non indiqués dans la documentation. Ces informations nous montrent clairement le rôle de l'héritage dans la constitution de la propriété des femmes hiérosolymites.

Ensuite, dans une autre optique, en examinant les noms des vendeuses et des acheteurs / acheteuses, nous constatons que seulement quatorze sur quatre-vingt-huit vendeuses ont vendu leurs biens à des proches tandis que les autres vendeuses ont vendu leurs propriétés aux individus appartenant à d'autres familles. Cela nous laisse croire que la question du prix et du marché rentrent dans les calculs de ces femmes et non seulement la question familiale.

Le troisième exemple : le positionnement des hommes par rapport au droit de propriété des femmes et de leur droit à disposer de leurs biens fonciers

Nous avons donc bien noté dans les deux parties précédentes que les femmes possèdent des biens par voie de l'héritage et par achat des propriétés foncières et ont le droit d'en disposer à leur guise. Nous avons vu aussi que ces droits ont permis aux femmes d'avoir une place quelquefois très importante dans la société et dans le milieu socio-économique et charitable bien qu'elles doivent le partager avec leurs proches (mari, frères, sœurs, etc.). Mais vient alors la question : est-ce que les composants masculins de la société ont accepté ce droit? S'il y a eu opposition, comment a-t-elle été exprimée? 
TABLEAU $\mathrm{N}^{\circ} 4 \quad$ Vente par des femmes hiérosolymites de leurs biens fonciers entre 1831-1841

\begin{tabular}{|c|c|c|c|c|c|c|}
\hline $\mathbf{N}^{\circ}$ & $\begin{array}{l}\text { Nom de la } \\
\text { vendeuse }\end{array}$ & Nom d'acheteur & $\begin{array}{l}\text { Type de } \\
\text { bien }\end{array}$ & $\begin{array}{l}\text { Source de la } \\
\text { propriété }\end{array}$ & Prix & Date \\
\hline 1 & $\begin{array}{l}\text { Fațûma al-Ḥâjj } \\
\text { Ḥasan }\end{array}$ & $\begin{array}{l}\text { Muhammad } \\
\text { Shu'ayb }\end{array}$ & $\begin{array}{l}1 \text { partie / } \\
\text { dâr }\end{array}$ & $\begin{array}{l}\text { Héritage de } \\
\text { son père }\end{array}$ & $?$ & $\begin{array}{l}1246 / \\
1831^{a}\end{array}$ \\
\hline 2 & Ruqayya al-'Arabî & Mușțafâ al-Iskâfî & $\begin{array}{l}4 \text { qîrâț / } \\
\text { dâr }\end{array}$ & Achat & 500 & $\begin{array}{l}1248 / \\
183^{b}\end{array}$ \\
\hline 3 & Fâțima al-Mașrî & $\begin{array}{l}\text { Ahmad } \\
\text { al-Qudbajî }\end{array}$ & $\begin{array}{l}20 \text { qîrâts / } \\
\text { dâr }\end{array}$ & $\begin{array}{l}\text { Héritage de } \\
\text { son mari }\end{array}$ & 1150 & $\begin{array}{l}1248 / \\
1833^{c}\end{array}$ \\
\hline 4 & Nasab al-Disûqî & $\begin{array}{l}\text { 'Abd al-Raḥmân } \\
\text { al-Khazantî }\end{array}$ & $\begin{array}{l}10 \text { qîrâț / } \\
\text { dâr }\end{array}$ & $\begin{array}{l}\text { Héritage de } \\
\text { son frère }\end{array}$ & 430 & $\begin{array}{l}125^{\mathrm{o}} / \\
1834^{\mathrm{d}}\end{array}$ \\
\hline 5 & Zînab al-Jâ'ûnî & $\begin{array}{l}\text { Aḥmad } \\
\text { al-Nashshâshibî }\end{array}$ & $\begin{array}{l}11 \text { qîrâțs / } \\
\text { khulû dâr }\end{array}$ & $\begin{array}{l}\text { Héritage de } \\
\text { sa mère }\end{array}$ & 1600 & $\begin{array}{l}125^{1 /} \\
1835^{\mathrm{e}}\end{array}$ \\
\hline 6 & $\begin{array}{l}\text { Nafîsa } \\
\text { al-Nashshâshîbî }\end{array}$ & Ḥasan al-Fityyânî & $\begin{array}{l}3 \cdot 5 \text { qîrâts / } \\
\text { four }\end{array}$ & $\begin{array}{l}\text { Héritage de } \\
\text { son père }\end{array}$ & 135 & $\begin{array}{l}125^{2 /} \\
183^{f}\end{array}$ \\
\hline 7 & $\begin{array}{l}\text { Ammûna } \\
\text { al-Nâbulsî }\end{array}$ & Șâliḥ al-Râzî & 1 dâr & $\begin{array}{l}\text { Héritage de } \\
\text { son ancien } \\
\text { mari }\end{array}$ & 2500 & $\begin{array}{l}1253 / \\
1837^{\mathrm{g}}\end{array}$ \\
\hline 8 & $\begin{array}{l}\text { Ghuṣûn } \\
\text { al-Dâwwûdî }\end{array}$ & Ḥasan al-Dajânî & $\begin{array}{l}2 \text { qîrâțs / } \\
\text { terrain }\end{array}$ & $\begin{array}{l}\text { Héritage de } \\
\text { son père }\end{array}$ & $6 o$ & $\begin{array}{l}1253 / \\
1838^{\mathrm{h}}\end{array}$ \\
\hline 9 & $\begin{array}{l}\text { Maḥbûba } \\
\text { al-'Alamî }\end{array}$ & $\begin{array}{l}\text { 'Abd Allâh } \\
\text { al-'Alamî }\end{array}$ & $\begin{array}{l}15 \text { qîrâts / } \\
\text { dukkân }\end{array}$ & $\begin{array}{l}\text { Héritage de } \\
\text { son père }\end{array}$ & 4833 & $\begin{array}{l}1255 / \\
1839^{i}\end{array}$ \\
\hline 10 & Ṣâliḥa al-Busțâmî & 'Issâ al-Busțâmî & $\begin{array}{l}6 \text { qîrâts / } \\
\text { dâr }\end{array}$ & $\begin{array}{l}\text { Héritage de } \\
\text { son mari }\end{array}$ & 400 & $\begin{array}{l}125^{6 /} \\
1840^{j}\end{array}$ \\
\hline
\end{tabular}

a Sijill 315, Dhû al-Hijja 1246 / 27 mai 1831, p. 4.

b Sijill 317, 21 Muḥarram 1248 / 20 juin 1832, p. 12.

c Sijill 317 , début Rabî̀ II 1248 / 18 août 1833, p. 36.

d Sijill 319, fin Muhararam 1250 / 8 juin 1834, p. 130.

e Sijill 319, 12 Rabî' II 1251 / 7 août 1835, p. 154.

f Sijill 320, milieu Rabî‘ I 1252 / 30 juin 1836, p. 103.

g Sijill 321, 10 Muharram 1253 / 16 avril 1837, p. 58.

h Sijill 321, 23 Dhû al-hijja 1253 / 20 mars 1838, p. 243.

i Sijill 322, 22 Ṣafar 1255 / 7 mai 1839, p. 200.

j Sijill 323, milieu Șafar 1256 / 18 avril 1840, p. 122. 
Pour répondre à ces questions, nous avons examiné dix registres des juges de Jérusalem qui couvrent la période de notre étude 1831-1841. Nous avons trouvé quinze procès déposés devant le juge de Jérusalem par des femmes hiérosolymites concernant la spoliation de leurs biens immobiliers où il est question de leurs droits de propriété contestés par des hommes provenant de leurs proches.

A l'analyse de ces quinze documents au sujet des litiges concernant les propriétés foncières à Jérusalem pendant la période de l'étude, nous constatons que dans la plupart des cas, il y a eu des conflits violents à la fois concrets concernant des spoliations diverses et une démarche abstraite de refus de l'idée que les femmes aient un droit de propriété. Plus encore, un refus du droit de la liberté de disposer des biens, par exemple, en le vendant à une personne n'ayant aucun lien familial ou religieux avec la famille. Dans plusieurs cas, nous remarquons que la femme a été obligée de déposer des plaintes auprès du juge de Jérusalem et de prendre un représentant (wakîl) qui défendait ses droits contre ses proches avec lesquels elle partageait la propriété ou contre d'autres spoliateurs. Pour bien montrer les détails de ces documents, nous proposons le tableau suivant $\mathrm{n}^{\circ} 5$.

TABLEAU $\mathrm{N}^{\circ} 5 \quad$ Les cas des litiges sur les droits de propriété des femmes, $183_{1-1841}$

\begin{tabular}{lll}
\hline $\mathrm{N}^{\circ}$ & Nom de la femme \\
& propriétaire
\end{tabular}

\begin{tabular}{|c|c|c|c|c|}
\hline 1 & $\begin{array}{l}\text { Khadîja } \\
\text { al-Tamîmî }^{\mathrm{a}}\end{array}$ & Balkir 'Awâd & 1 & Dâr \\
\hline 2 & $\begin{array}{l}\text { Sa'âda } \\
\text { al-Turjumân }{ }^{\text {b }}\end{array}$ & $\begin{array}{l}\text { al-Shaykh } \\
\text { Sulaymân }\end{array}$ & $\begin{array}{l}\text { Ses partenaires } \\
\text { de l'héritage de } \\
\text { son mari }\end{array}$ & 1 partie d'une dâr \\
\hline 3 & 'Â'isha Hadûtac & $\begin{array}{l}\text { 'Abd al-Raḥmân } \\
\text { Hadûta }\end{array}$ & Son cousin & 1 partie d'une dâr \\
\hline 4 & $\begin{array}{l}\text { Ṣâliḥa Abû } \\
\text { Ḥasna }^{\mathrm{d}}\end{array}$ & Ḥusayn al-Fityânî & l & 1 dukkân \\
\hline 5 & $\begin{array}{l}\text { Khadîja Ṣâliḥa } \\
\text { al-Raqța }^{\mathrm{e}}\end{array}$ & $\begin{array}{l}\text { Les filles de } \\
\text { Sulaymân al-Sa dî }\end{array}$ & 1 & 1 partie d'une dâr \\
\hline 6 & $\begin{array}{l}\text { Zînab `Uthmân } \\
\text { al-Jîțânf }\end{array}$ & Ḥasan Bîk al-Jîtân & Son neveu & $\begin{array}{l}\text { Revenus de waqf } \\
\text { de son grand père }\end{array}$ \\
\hline 7 & $\begin{array}{l}\text { Hasab ‘Abd Allâh } \\
\text { Bîk 'Aqilg }\end{array}$ & Aḥmad Bîk 'Aqil & Son cousin & $\begin{array}{l}\text { Héritage de son } \\
\text { père }\end{array}$ \\
\hline
\end{tabular}




\section{$\mathrm{N}^{\circ} \quad$ Nom de la femme Nom du spoliateur Nature des liens Type de bien-fonds propriétaire}

\begin{tabular}{|c|c|c|c|c|}
\hline 8 & $\begin{array}{l}\text { Nafîsa Bîk } \\
\text { al-Za 'îm h }\end{array}$ & D’autres héritiers & Ses cousins & $\begin{array}{l}\text { Héritage de son } \\
\text { père }\end{array}$ \\
\hline 9 & $\begin{array}{l}\text { Fâtṭuma Șâlih } \\
\text { al-'Ajamîi }^{\text {al }}\end{array}$ & $\begin{array}{l}\text { Ibrâhîm Ấghâ, } \\
\text { gérant de waqf de } \\
\text { son grand père }\end{array}$ & l & $\begin{array}{l}\text { Revenue de waqf } \\
\text { de son grand père }\end{array}$ \\
\hline 10 & $\begin{array}{l}\text { Fâțima Mûsâ } \\
\text { Najim }\end{array}$ & Ibrâhîm Ghûshâ & Un locateur & $\begin{array}{l}\text { Le loyer de sa } \\
\text { maison }\end{array}$ \\
\hline 11 & $\begin{array}{l}\text { Ammûna Khalîl } \\
\text { al-'Akîlîkk }\end{array}$ & Anțûnî al-Rûmî & Son partenaire & $\begin{array}{l}\text { Sa partie d'une } \\
\text { maison }\end{array}$ \\
\hline 12 & $\begin{array}{l}\text { Tarafanda Mûsa } \\
\text { al-Khalîlî̀ }^{1}\end{array}$ & $\begin{array}{l}\text { 'Umar Qassâb } \\
\text { Bâshî }\end{array}$ & 1 & $\begin{array}{l}\text { Son droit dans } \\
\text { l'héritage de sa } \\
\text { mère }\end{array}$ \\
\hline 13 & $\begin{array}{l}\text { Șafiyya } \\
\text { Muhạmmad } \\
\text { Marash et sa fille } \\
\text { Ấmîna Karâmm }\end{array}$ & $\begin{array}{l}\text { Muhammad } \\
\text { Ibrâhîm Karâm }\end{array}$ & $\begin{array}{l}\text { La femme de } \\
\text { son père et sa } \\
\text { fille }\end{array}$ & $\begin{array}{l}\text { Leurs droits dans } \\
\text { l'héritage de son } \\
\text { mari, une maison }\end{array}$ \\
\hline 14 & $\begin{array}{l}\text { 'Â'isha Musțafa } \\
\text { al-Bayrûtîn }\end{array}$ & $\begin{array}{l}\text { Ibrâhîm Musțafâ } \\
\text { al-Bayrûtî }\end{array}$ & Son frère & $\begin{array}{l}\text { La gestion de } w a q f \\
\text { de son père }\end{array}$ \\
\hline 15 & $\begin{array}{l}\text { Khânum Hasan } \\
\text { al-'Akârî̀ }\end{array}$ & $\begin{array}{l}\text { 'Abd Allâh Mûsâ } \\
\text { al-'Akârî }\end{array}$ & Un héritier & $\begin{array}{l}\text { Sa partie d'une } \\
\text { maison }\end{array}$ \\
\hline
\end{tabular}

a Sijill 315, 3 Muḥarram 1247 / 14 juin 1831, p. 8.

b Sijill 315, début Rahab 1247 / 6 décembre 1831, p. 128.

c Sijill 320, 10 Shawwâl 1251 / 29 janvier 1836, p. 24.

d Sijill 320, 15 Shawwâl 1251 / 3 février 1836, p. 29.

e Sijill 320, 10 Shawwâl $125^{1}$ / 29 janvier 1836, p. 30.

f Sijill 321, 13 Rabî́ I 1253 / 17 juin 1837, p. 74.

g Sijill 321, début Muharram 1253 / avril 1837, p. 87.

h Sijill 321, fin Rabî‘ I 1253 / 3 août 1837, p. 97.

i Sijill 321, fin Rabî̀ I 1253 / 3 août 1837, p. 102.

j Sijill 321, 10 Jumâdâ I 1253 / 11 septembre 1837, p. 161.

k Sijill 322, milieu Rabî̀ I 1254 / 8 juin 1838, p. 39.

l Sijill 322, 25 Jumâda I 1254 / 16 août 1838, p. 71.

m Sijill 322, fin Rajab 1254 / 19 octobre 1838, p. 106.

n Sijill $_{324}$, 10 Jumâda I 1254 / 10 juillet 1840, p. 8.

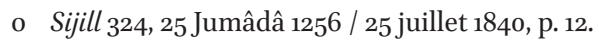


Ce tableau montre clairement que le droit de propriété des femmes hiérosolymites n'a pas été toujours respecté, des femmes ont bien été spoliées par leurs proches. Onze parmi les quinze cas mentionnés dans le tableau sont des cas de spoliation menés par des proches - frère, neveu, cousin, héritiers, etc., qui montre clairement le statut de propriété des femmes était moins considéré à l'intérieur de la structure familiale par rapport au droit donné dans la jurisprudence musulmane.

Il faut signaler que la période de cette étude n'était pas un cas exceptionnel pour cette question; nous trouvons, en effet, ce phénomène à Jérusalem durant toute la période du XIX $\mathrm{X}^{\mathrm{e}}$ siècle où des cas similaires sont à constater. Nous avons choisi quelques exemples pour illustrer ces faits. A la fin du mois Dhû al-Qa da 1218 / 12 mars 1804, Muștafâ Ashraf Hijîij a porté plainte auprès du juge du tribunal de Jérusalem quand il s'est aperçu que le commerçant Sulaymân Quțtîna était installé dans les biens appartenant à sa femme Șafiyya Șaliḥ Ḥijîj. Cette dernière a confirmé qu'elle avait vendu ce bien mais son mari a persisté à contester cette vente comme illégale. Quant au juge, après avoir entendu les deux parties ainsi que les témoins, puis après avoir examiné le contrat de vente, il a déclaré que la plainte était irrecevable et a confirmé la légalité du contrat de vente. Il a ensuite donné l'autorisation pour que le commerçant ait l'usage de ce bien sans que le mari ne s'y oppose ${ }^{17}$.

Un autre exemple : au milieu du mois Dhû al-Hijja 1219 /17 mars 1805, 'Umar al-Jâ' ûnî a porté plainte contre l'acquéreur d'une maison située dans le quartier chrétien vendue à un chrétien Anțûn Bin Ḥannâ par son épouse Nasab Șaliḥ al-Jâ'ûnî sous prétexte que cette vente d'un montant de trois cent cinquante zolța valait, selon les valeurs du marché, mille zolța. Il demandait que l'acquéreur paie la différence, sinon la vente serait annulée. Devant le juge, sa femme a déclaré toutefois que la vente était légale et qu'elle avait reçu le prix demandé et l'acheteur n'avait donc rien à payer en plus. Après enquête, le juge a donné sa décision et a débouté le mari de sa plainte ${ }^{18}$.

Ce scenario n'est pas toujours le même, à savoir un cas où le mari attaque l'usage fait du droit foncier par sa femme. En effet, nous trouvons des cas où un mari est amené à défendre le bien foncier de sa femme qui a été spoliée par son propre frère. A titre d'exemple, au début du mois Jumâdâ I 1223 / 25 juin 1808, 'Abd Allâh Shakkî Makkiyya a déposé une plainte devant le juge de Jérusalem contre Dâwûd 'Alî Hijijîj, le frère de sa femme. Dans sa plainte, il dit que sa femme, Laț̂fa 'Alî Hiijîjj a hérité de son père, avec son frère, d'un four situé dans le quartier chrétien mais que le frère n'ait pas accepté ni le principe

17 Sijill 287, fin Dhû al-Qa'da 1218 / 12 mars 1804, p. 6.

18 Sijill 287, milieu Dhû al-Hijja 1219 /17 mars 1805, p. 161. 
du partage ni l'usage. Le juge, après examen des faits, a trouvé un testament qui démontre bien le droit de Laṭ̂fa à posséder ce four autant que son frère. Il a pris la décision de demander au frère les arriérés dûs pour la location du four ${ }^{19}$.

\section{Conclusion}

Cette contribution a tenté de démontrer la place importante de la femme dans la société civile musulmane en étudiant son droit de posséder la propriété et à la liberté d'en disposer, protégée par le droit religieux, le fiqh, comme nous l'avions pu constater lors de cette étude par les jugements en leur faveur inscrits dans les registres du tribunal. En effet, nous avons vu que les musulmanes de Jérusalem de la période égyptienne 1831-1841 accèdent aux propriétés par l'achat de celles-ci ou par voie de l'héritage; en outre, nos documents montrent qu'elles avaient fait fructifier leurs biens fonciers, à savoir des maisons mais aussi des commerces. Cette étude a également démontré qu'elles pouvaient habiter leurs propriétés bâties ou les vendre ou, enfin, les transformer en waqf. Nous avons remarqué que tous les waqfs fondés par des musulmanes à cette période ont été des waqfs familiaux. Nous n'avons trouvé aucun waqf charitable, c'est-à-dire que les waqfs de ces femmes n'étaient fondés qu'en leur faveur, cette procédure étant aussi un moyen de protéger leurs biens contre la possible spoliation des proches, le waqf étant protégé par le droit musulman.

Pour conclure, le milieu familial musulman à Jérusalem, sans toutefois le généraliser, acceptait le principe d'un droit de propriété et le droit de l'usage pour les femmes et pour les hommes. Or, dans la pratique, comme nous avions montré dans cette étude, il y avait des familles hiérosolymites qui n'ont pas accepté, dans la pratique, le droit accordé aux femmes dans la jurisprudence musulmane à l'égard d'une propriété, ni le droit de l'usage car non seulement les frères n'ont pas accepté, dans des cas cités ci-dessus, le partage de l'héritage du père avec leurs sœurs mais aussi le fait que le mari refusait à sa femme le droit de disposer de ses biens hérités de son père ou d'un ancien mari. Dans ce cas, la musulmane est doublement défavorisée. D'une part, le droit religieux en islam ne lui ait pas accordé un statut d'égalité vis-à-vis de l'héritage de propriété avec l'homme et, d'autre part, le milieu familial n'a pas toujours respecté leurs droits accordé par le fiqh. Ces observations, établies à partir de notre étude réalisée auprès des documents de registres du tribunal ottoman de Jérusalem de 1831 à 1841 n'a rien d'exceptionnel, ces pratiques sont toujours appliquées actuellement en Palestine.

19 Sijill 292, début Jumâdâ I 1223 / 25 juin 1808, p. 22. 


\section{Bio}

Musa Sroor obtained his doctoral diploma in 2005 from Aix-Marseille University, France, under the supervision of Randi Deguilhem. Since then, he has been associate professor at Birzeit University, Palestinian Territories, where he teaches contemporary history and is currently director of the Department of History and Archeology and director of the master's program in history. His research mostly concerns the question of waqf and the socioeconomic history of Jerusalem during the Ottoman period.

\section{Bibliographie}

\section{Documents d'archives}

Le centre d'archives d'Abû Dîs, Territoires palestiniens

Sijills : Registres du tribunal religieux de Jérusalem

Sijill 270, 964 / 1557

Sijill 287, 1218 /1804

Sijill 292, 1223 / 1808

Sijill 315, 1246-1247 / 1831

Sijill 316, 1247-1248 / 1831-1832

Sijill 317, 1248-1249 / 1832-1833

Sijill 318, 1249-1250 / 1833-1834

Sijill 319, 1250-1251 / 1834-1835

Sijill 320, 1251-1252 / 1836-1837

Sijill 321, 1252-1253 / 1836-1837

Sijill 322, 1253-1254/1837-1838

Sijill 323, 1254-1256 / 1839-1840

Sijill 324, 1256-1257 / 1840-1841

\section{Travaux publiés}

'Abd al-Mahdî, 'Abd al-Jalîl, 1981, al-Madâris fî bayt al-maqdis fî al-'asrayn al-ayûb̂̂ wa-'l-mamlûkî, Amman, Maktabat al-Aqșâ, vol. 1.

Abû Bakr, Amîn, 1996, Mulkiyyat al-arâdî fî mutasarrifiyyat al-Quds 1858-1917, Amman, Mu'asasat 'Abd al-Hamîd Shûmân. 
Ben Achour, Mohamed, 1992, « Le habous ou waqf: l'institution juridique et la pratique tunisoise", in Sophie Ferchiou, dir., Hasab wa nasab. Parenté, alliance et patrimoine en Tunisie, Paris, Editions du CNRS, p. 51-78.

Deguilhem, Randi, 1986, History of waqf and case studies from Damascus in the late Ottoman and French mandatory times, thèse de doctorat, New York University, Ann Arbor Mich, UMI, University Microfilms.

Deguilhem, Randi, 1995a, «Approche méthodologique d'un fonds de waqf: deux registres de Shariâ du XIX ${ }^{\mathrm{e}}$ siècle à Damas », in Randi Deguilhem, dir., Le waqf dans l'espace islamique, outil de pouvoir socio-politique, Damas, Institut Français d'Etudes Arabes de Damas (IFEAD), p. 45-70.

Deguilhem, Randi, 1995b, « Naissance et mort du waqf damascain de Ḥafiẓa Hânûm al-Mûrahli (1880-1950)» in Randi Deguilhem, dir., Le waqf dans l'espace islamique, outil de pouvoir socio-politique, Damas, IFEAD, p. 203-25.

Denoix, Sylvie, 1995, « Pour une exploitation d'ensemble d'un corpus : les waqfs mamelouks du Caire », in Randi Deguilhem, dir., Le waqf dans l'espace islamique, outil de pouvoir socio-politique, Damas, IFEAD, p. 29-44.

Kallâf, 'Abd al-Wahhâb, 1946, Ahkâm al-waqf, Le Caire.

Manna, 'Adel, 1986, "The Sijill as Source for the Study of Palestine during the Ottoman Period with Special Reference to the French Invasion", in David Kushner, ed., Palestine in the late Ottoman Period, : Political, Social and Economic Transformation, Jerusalem, Yad Izhak Ben-Zvi / Leiden, Brill, p. 351-62.

Al-Mar'a al-Filațtîniyya wa-'l-Mîrâth, Markaz al-mar'a li-'l-irshâd al-qânûnî wa-'lijtimâî̀, Filastîn, 2014.

Pascual, Jean-Paul, 1990, « Du notaire au propriétaire en passant par l'expert : descriptions de la 'maison' damascène au XVII ${ }^{\mathrm{e}}$ siècle», extrait de L'Habitat traditionnel dans les pays musulmans autour de la Méditerranée : l'histoire et le milieu, Le Caire, Institut français d'archéologie orientale, vol. II, p. 387-403.

Safi, Khaled, 2004, The Egyptian Rule in Palestine 1831-1840: A Critical Reassessment, Berlin, Bensch \& Buch Verlag.

Salameh, Khadr, 2000, "Aspects of the Sijill of the Shari'a Court in Jerusalem", in S. Auld et Yusuf Natsheh, éd., Ottoman Jerusalem. The Living City: 1517-1917, London, Altajir World of Islam Trust, 103-45.

Saliba, Sabine, 2004, «Waqf et gérance familiale au Mont Liban à travers l'histoire du couvent maronite de Mar Challita Mouqbès (XVII ${ }^{\mathrm{e}}-\mathrm{XIX}^{\mathrm{e}}$ siècles) », in Randi Deguilhem et Abdelhamid Henia, coord., Les fondations pieuses (waqf) en Méditerranée : enjeux de société, enjeux de pouvoir, Koweït, La Fondation Publique des Awqaf du Koweït, p. 99-131.

Sroor, Musa, 2010, Fondations pieuses en mouvement: de la transformation du statut de propriété des biens waqfs à Jérusalem (1858-1917), Damas, Institut du Proche-Orient 
(IFPO) et Institut de Recherches et d'Etudes sur le Monde arabe et Musulman (IREMAM).

Sroor, Musa, 2005 (winter), "Jerusalem's Islamic Archives: Sources for the question of the waqf in the Ottoman period", Jerusalem Quarterly, $\mathrm{n}^{\circ}$ 22-23, p. 80-6.

Stephan, H. F., 1944, "An Endowment Deed of Khasseki Sultan, dated 24th May 1552", Quarterly of the Department of Antiquities in Palestine, X, p. 170-94.

Yediyildiz Bahaeddin, 1985, Institution du vaqf au XVIII e siècle en Turquie : étude sociohistorique, Ankara, Ministère de la Culture. 